\title{
BMJ Open Axillary versus innominate artery cannulation for antegrade cerebral perfusion in aortic surgery: design of the Aortic Surgery Cerebral Protection Evaluation (ACE) CardioLink-3 randomised trial
}

Vinay Garg, ${ }^{1}$ Mark D Peterson, ${ }^{2,3}$ Michael WA Chu, ${ }^{4}$ Maral Ouzounian, ${ }^{3,5}$ Roderick GG MacArthur, ${ }^{6}$ John Bozinovski, ${ }^{7}$ Ismail El-Hamamsy, ${ }^{8} \mathrm{~F}$ Victor Chu, ${ }^{9}$ Ankit Garg, ${ }^{1}$ Judith Hall, ${ }^{10}$ Kevin E Thorpe, ${ }^{10,11}$ Natasha Dhingra, ${ }^{2}$ Hwee Teoh, ${ }^{12,13}$ Thomas R Marotta, ${ }^{14,15}$ David A Latter, ${ }^{2,3}$ Adrian Quan, ${ }^{12}$ Muhammad Mamdani, ${ }^{15,16,17,18,19}$ Peter Juni, ${ }^{10,16}$ C David Mazer, ${ }^{20,21}$ Subodh Verma ${ }^{3,12,22}$

To cite: Garg V, Peterson MD, Chu MWA, et al. Axillary versus innominate artery cannulation for antegrade cerebral perfusion in aortic surgery: design of the Aortic Surgery Cerebral Protection Evaluation (ACE) CardioLink-3 randomised trial. BMJ Open 2017;7:e014491. doi:10.1136/ bmjopen-2016-014491

- Prepublication history and additional material are available. To view these files please visit the journal online (http://dx.doi.org/ 10.1136/ bmjopen-2016-014491)

Received 4 November 2016 Revised 13 April 2017

Accepted 21 April 2017

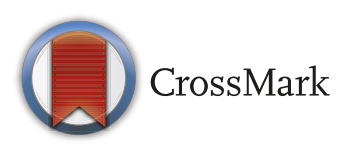

For numbered affiliations see end of article.

Correspondence to

Dr Subodh Verma;

vermasu@smh.ca

\section{ABSTRACT}

Introduction Neurological injury remains the major cause of morbidity and mortality following open aortic arch repair. Systemic hypothermia along with antegrade cerebral perfusion (ACP) is the accepted cerebral protection approach, with axillary artery cannulation being the most common technique used to establish ACP. More recently, innominate artery cannulation has been shown to be a safe and efficacious method for establishing ACP. Inasmuch as there is a lack of high-quality data comparing axillary and innominate artery ACP, we have designed a randomised, multi-centre clinical trial to compare both cerebral perfusion strategies with regards to brain morphological injury using diffusion-weighted MRI (DWMRI).

Methods and analysis 110 patients undergoing elective aortic surgery with repair of the proximal arch requiring an open distal anastamosis will be randomised to either the innominate artery or the axillary artery cannulation strategy for establishing unilateral ACP during systemic circulatory arrest with moderate levels of hypothermia. The primary safety endpoint of this trial is the proportion of patients with new radiologically significant ischaemic lesions found on postoperative DW-MRI compared with preoperative DW-MRI. The primary efficacy endpoint of this trial is the difference in total operative time between the innominate artery and the axillary artery cannulation group.

Ethics and dissemination The study protocol and consent forms have been approved by the participating local research ethics boards. Publication of the study results is anticipated in 2018 or 2019. If this study shows that the innominate artery cannulation technique is noninferior to the axillary artery cannulation technique with regards to brain morphological injury, it will establish the innominate artery cannulation technique as a safe and
Strengths and limitation of this study

Multi-centre randomised controlled trial.

- Rigorous design to address an important unanswered question with clinically relevant primary and secondary outcomes.

- Strong research team with experts in thoracic aortic surgery and cerebral perfusion techniques.

- Study population limited to elective procedures of the ascending aorta and proximal arch, excluding aortic dissections and emergency operations.

- Patient outcomes only followed to 3 months.

- Study requires surgeons skilled in both cannulation techniques.

potentially more efficient method of antegrade cerebral perfusion in aortic surgery.

Trial registration number NCT02554032.

\section{INTRODUCTION}

Thoracic aortic aneurysms are associated with substantial morbidity and mortality. Accordingly, aortic surgery is common, with approximately 200000 surgical cases annually worldwide. ${ }^{1-3}$ The natural history of unrepaired aortic aneurysms is poor, with a high incidence of aortic dissection, rupture and death. 124

Surgery involving the ascending aorta and aortic arch is complex, and several techniques have been developed to safely interrupt or modify the circulation to the brain. Despite advances in cannulation, 
cerebral perfusion and temperature management, neurological injury remains the most dreaded complication of aortic arch surgery. ${ }^{35}$ Manifestations of such injury may range from fatal or severe/irreversible injury to milder transient ischaemic attack (TIA), or neurocognitive injury. ${ }^{2356}$

Current practice for proximal aortic arch surgery with regards to neuroprotection strategies include deep hypothermic circulatory arrest (DHCA) alone, retrograde cerebral perfusion with DHCA, antegrade cerebral perfusion (ACP) with DHCA and moderate hypothermic circulatory arrest with ACP. ${ }^{6}$ In addition to moderate hypothermia, ACP via right axillary artery cannulation has become a preferred approach for cerebral protection during aortic surgery. ${ }^{7}$ Although ACP via the axillary artery has been shown to improve survival and neurological outcomes after aortic aneurysm repair compared with retrograde cerebral perfusion, there are several associated risks. ${ }^{6-14}$ The axillary approach increases the risk of brachial plexus injury, seromas, arm hyper-perfusion and limb ischaemia, and it requires additional surgical dissection, increasing the total operative time required, particularly in patients with obesity or challenging anatomy. ${ }^{10}{ }^{15}$ A novel approach for delivering ACP via cannulation of the innominate artery has recently emerged (figure 1) ${ }^{16}$ First devised by Banbury and colleagues in 2000, several retrospective studies and case series have shown innominate artery cannulation to be relatively safe with a low rate of surgical mortality and neurological injury. ${ }^{10-22}$ A retrospective analysis comparing innominate artery cannulation with axillary artery cannulation showed no significant differences in neurological complications. ${ }^{15}$ However, data evaluating innominate artery cannulation are confounded by potential selection bias with respect to the complexity of patients chosen for each strategy. Although worldwide many surgeons have adopted the innominate artery cannulation strategy in favour of the axillary artery strategy, there are no randomised trial data to help objectively evaluate safety and efficacy of this technique. Given the grave consequences of inappropriate and/or inadequate cerebral protection, a randomised trial to compare each surgical strategy is needed. We describe herein the protocol for the prospective Aortic Surgery Cerebral Protection Evaluation (ACE) CardioLink-3 randomised trial that has been designed to establish the efficacy and safety of innominate artery cannulation versus axillary artery cannulation for ACP in patients undergoing proximal aortic arch surgery with hypothermic circulatory arrest.

\section{Study Purpose}

The purpose of this trial is to compare innominate artery cannulation to axillary artery cannulation as a means of cerebral protection during moderate hypothermic circulatory arrest in subjects undergoing surgery for aneurysms of the ascending aorta and proximal arch. The primary safety objective of this study is to determine whether the innominate artery cannulation technique is non-inferior to the axillary artery cannulation technique for establishing ACP with regards to brain morphological injury on diffusion-weighted magnetic resonance imaging (DW-MRI). The primary efficacy objective is to determine if the innominate artery technique is superior to the axillary artery technique with respect to surgical operative time.

The secondary objectives are to determine whether innominate artery cannulation for ACP is non-inferior to axillary artery cannulation with regards to postoperative 30 day mortality, and clinical and biomarker evidence of neurological injury and cognitive dysfunction.

\section{METHODS}

\section{Study Design Summary}

This is a multi-centre, two-arm randomised controlled, non-inferiority trial comparing a novel strategy for establishing ACP via innominate artery cannulation versus traditional axillary artery cannulation in patients 18 years and older undergoing elective repair of the ascending aorta and proximal arch requiring moderate hypothermic circulatory arrest and an open distal anastomosis. Patients undergoing surgery for aortic dissection, emergent or urgent operations, and total arch repair will be excluded. Furthermore, patients who cannot undergo MRI, or are using an investigational drug/device at the time of enrolment, or are a part of another clinical trial will also be excluded. Patients will be eligible for randomisation regardless of the proximal extent of the aortic repair (ie, root replacement vs. valve repair/replacement vs. supracoronary aortic repair). The patient will only remain in the study if the surgeon confirms the technical acceptability of either technique, as to allow for safe randomisation. Patients will then be randomly allocated 1:1 to undergo either the innominate or axillary cannulation strategy (figure 2). All participating centres are academic hospitals within Canada who have expertise in cardiac and aortic surgery (see online supplementary appendix 1 ). The trial has been registered at ClinicalTrials.gov (Identifier: NCT02554032).

\section{Randomisation}

Patients will be randomised in a 1:1 ratio, stratified by surgical centre, on the day of the scheduled ascending aorta operation to either the innominate artery cannulation strategy or the axillary artery cannulation strategy for establishing ACP during hypothermic circulatory arrest. The randomised assignments will be generated on a computer by a study statistician and will employ random permuted blocks of varying sizes. Randomisation will be centralised, web-based and generated by the Applied Health Research Center at the Li Ka Shing Knowledge Institute of St. Michael's Hospital. 
A

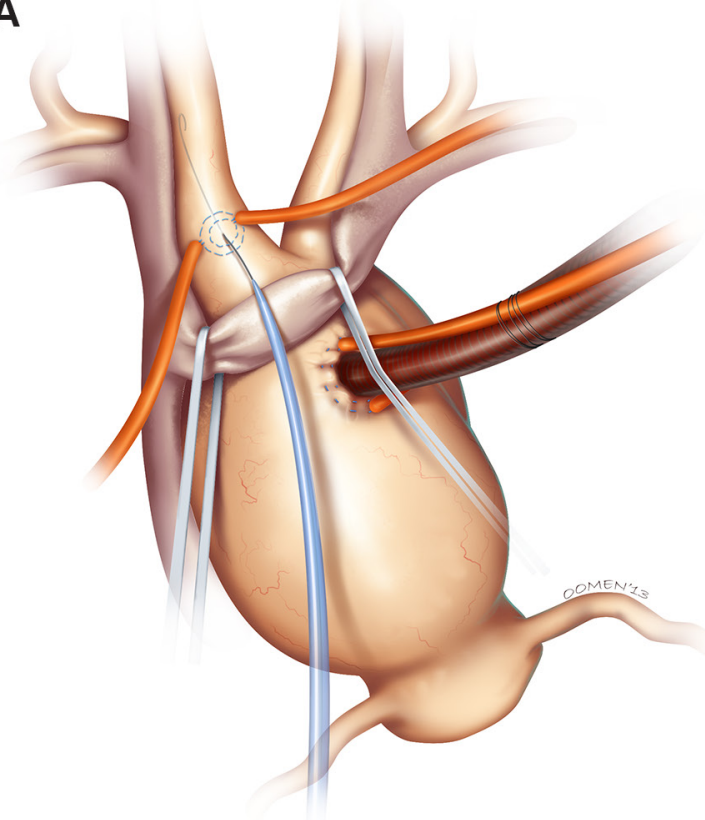

B

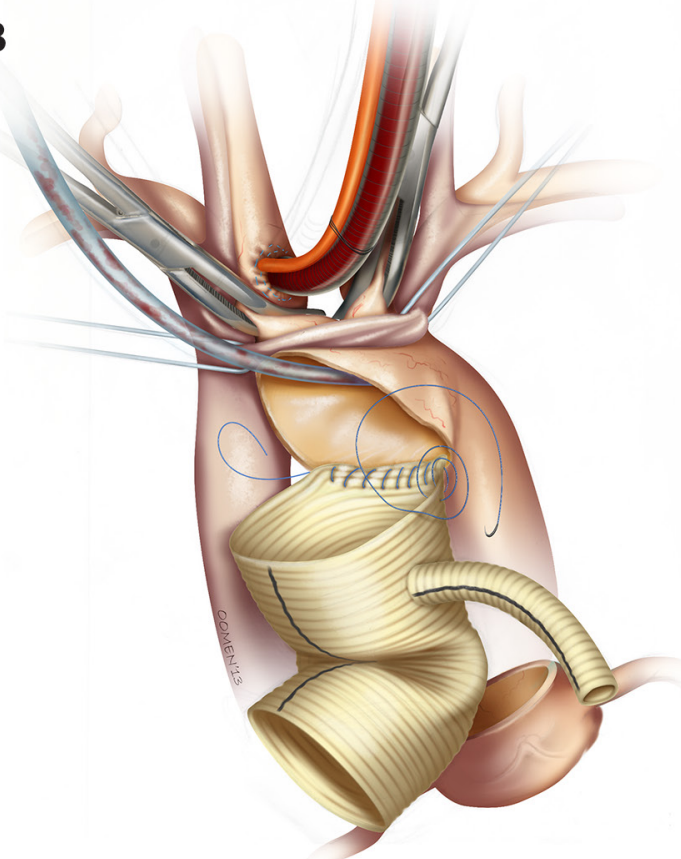

C

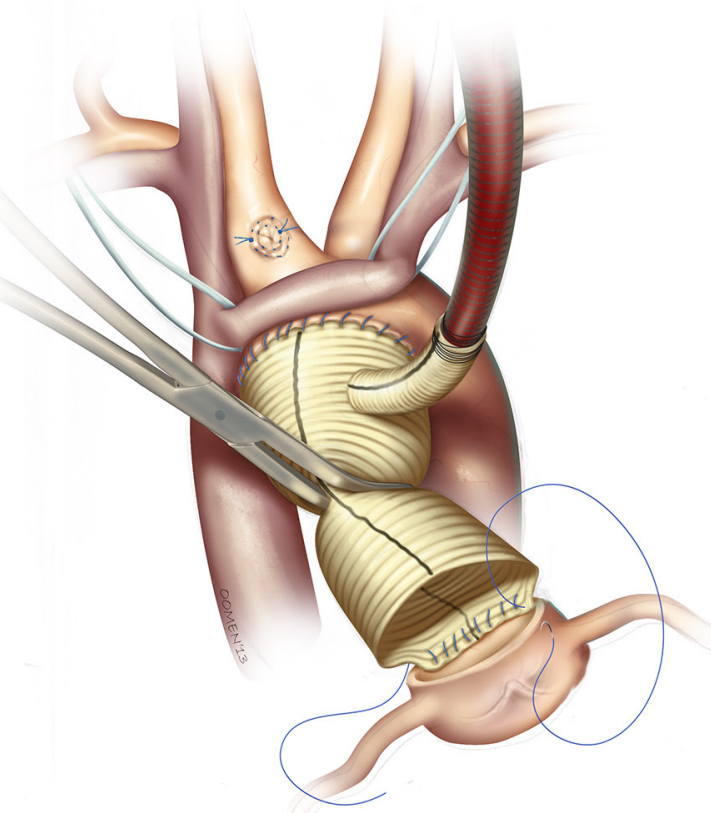

Figure 1 Surgical technique used in innominate artery cannulation (with permission from Garg et al). ${ }^{16}(\mathrm{~A})$ The innominate vein is isolated, retracted and the base of the innominate artery mobilised. Two purse string sutures are placed on the anterior wall of the proximal innominate artery before dilators and the cannula are inserted over a guide wire. (B) Antegrade cerebral protection is initiated by clamping the base of the innominate artery and connecting the afferent limb of the cardiopulmonary bypass circuit to the 14 or $16 \mathrm{Fr}$ cannula. (C) After the distal anastamosis is completed, antegrade cerebral protection is discontinued, and the aortic line is connected to the $8 \mathrm{~mm}$ side limb. The graft is clamped distally and full cardiopulmonary bypass is resumed. Proximal reconstruction is performed.

\section{Surgical strategy}

The surgical strategy will be standardised among the enrolling aortic surgeons/centres.

Innominate artery cannulation

After median sternotomy and systemic heparinisation, the distal aneurysmal ascending aorta will be cannulated using a standard 7 or $8 \mathrm{~mm}$ arterial cannula. Standard central venous cannulation is employed and cardioplegia management is left at the discretion of the individual surgeons; cardiopulmonary bypass (CPB) and systemic cooling will be initiated. During the cooling phase, the ascending aorta and proximal arch 


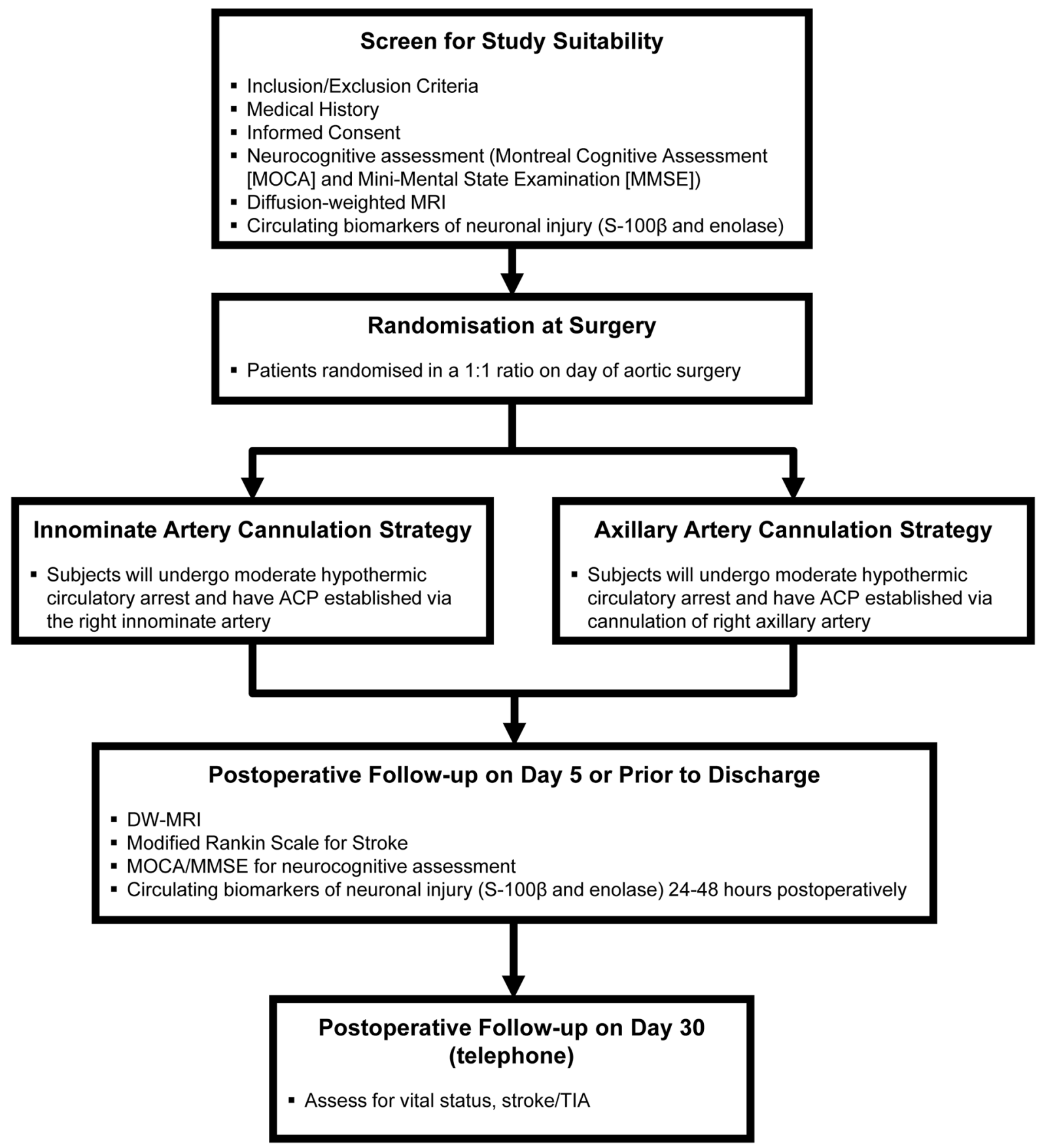

Figure 2 Study schematic of the ACE CardioLink-3 randomised trial.

will be mobilised, the innominate vein will be isolated and gently dissected, and the base of the innominate artery will be exposed. Two purse string sutures of 4-0 polypropylene will be placed on the anterior wall of the proximal innominate artery. Once the nasopharyngeal temperature is approximately $26^{\circ} \mathrm{C}$, the innominate artery will be cannulated with a 0.035 -inch $\mathrm{J}$ wire and sequentially dilated using $8 / 10 \mathrm{~F}$ and $12 / 14 \mathrm{~F}$ dilators. A $14 \mathrm{~F}$ or $16 \mathrm{~F}$ cannula with side perforations for gentle dispersion of the perfusate will then be inserted over a guide wire. The period of circulatory arrest with ACP will be initiated by clamping the base of the innominate artery and connecting the afferent limb of the CPB circuit to the cannula using a $3 / 8$ to $1 / 4$ connector. Moderately cold blood $\left(24^{\circ} \mathrm{C}-26^{\circ} \mathrm{C}\right)$ will be delivered at a flow rate of $10-12 \mathrm{~mL} / \mathrm{kg} / \mathrm{min}$ to achieve a right brachial pressure of $50-70 \mathrm{~mm} \mathrm{Hg}$. The distal anastamosis will be performed as either a bevelled hemiarch or an end-to-end anastamosis using a woven polyester graft with an $8 \mathrm{~mm}$ side limb. Once the distal anastamosis is complete, the aortic line will be connected to the side limb of the graft, air will be flushed from the graft, the graft clamped and full CPB will be resumed. At that time, ACP will be discontinued. Following 5 min at $26^{\circ} \mathrm{C}$, rewarming will resume and the proximal reconstruction will be completed. ${ }^{16}$ 
Axillary artery cannulation

Patients randomised to the control group will undergo axillary artery cannulation as routinely performed. The details of this operation have been previously described. ${ }^{15}$

\section{Outcomes}

\section{Primary outcome}

The primary safety endpoint of this trial is the proportion of patients with new radiologically significant ischaemic lesions. Two independent neuroradiologists who are blinded to treatment assignment will adjudicate this primary endpoint. New radiologically significant ischaemic lesions will be defined as severe white matter injury or an infarction involving the basal ganglia, thalamus or internal capsule or a large hemispheric infarction present on the postoperative DW-MRI. Each patient will have a preoperative DW-MRI to serve as the baseline study for comparison, to ensure the highest quality data. Severe white matter injury will be defined as six or more punctate lesions, or two or more lesions of $4 \mathrm{~mm}$ or larger size. ${ }^{5}$ We have included the size of the ischaemic lesions as a defining criterion for severity as the volume of ischaemic lesions is a known independent predictor of stroke outcome. ${ }^{23}{ }^{24}$ Furthermore, it has been shown that the number of DW-MRI lesions is only likely to be clinically relevant if the individual lesion is large. ${ }^{525} 26$

The primary efficacy endpoint of this trial is the difference in total operative time between the innominate artery cannulation group and the axillary artery cannulation group.

\section{Secondary outcomes}

Differences in the following secondary outcomes between both surgical strategies will be assessed:

1. 30 day all-cause postoperative mortality.

2. Postoperative stroke or TIA in hospital and at 30 days.

3. Neurocognitive dysfunction at postoperative day 4 and at 3 month follow-up as assessed by the MiniMental State Examination (MMSE) and Montreal Cognitive Assessment (MOCA) tests. Both the MOCA and MMSE have been validated for cognitive screening in patients with cerebrovascular disease. $^{27-29}$

4. DW-MRI parameters including number of total ischaemic lesions, total ischaemic volume of lesions, single lesion volume, watershed injury, intraparenchymal haemorrhage, cerebral haemorrhage, intraventricular haemorrhage, subdural haemorrhage, subarachnoid haemorrhage and partial cerebral sinovenous thrombosis.

5. Preoperative and postoperative serum levels of circulating biomarkers of neuronal injury (S-100 $\beta$ and neuron-specific enolase, NSE).

6. Postoperative sepsis, delirium, seizure, encephalopathy, atrial fibrillation, postoperative myocardial infarction (MI) and reoperations.
7. Total length of ICU stay, and number of hours of required intubation and ventilation.

8. Seroma, brachial plexus injury, reduced arm mobility and pain, and arm ischaemia.

9. Total CPB time, total cross-clamp time, total systemic circulatory arrest time, total ACP time, total cooling time (minutes), rewarming time, nadir nasopharyngeal temperature $\left({ }^{\circ} \mathrm{C}\right)$, postoperative haemoglobin, perioperative red blood cell (RBC) transfusion, perioperative inotrope or vasopressor use.

10. Cerebral oximetry will be monitored intraoperatively using bilateral non-invasive near-infrared spectroscopy. The following data will be evaluated: baseline $\mathrm{O}_{2}$ saturation while awake, total number of desaturations, that is, $<20 \%$ of baseline, the total area under the curve of percent saturation over time below $20 \%$ of baseline, minimal value reached and the maximum change from baseline values.

\section{Data Collection}

\section{Preoperative}

The screening visit will occur at the time of the initial appointment where the patient will be seen by the cardiac surgeon for evaluation and consideration of aortic surgery. Patients who are booked for elective ascending aortic surgery with a planned open distal anastamosis and moderate hypothermic circulatory arrest will be assessed for eligibility in the clinical trial. Study co-ordinators will review the study with potential participants and, on agreement to participate, obtain informed written consent.

The following baseline information will then be collected at either this initial visit or at the time of the preoperative anaesthesia clinic visit:

\section{Clinical information}

Demographics (age, sex), medical history including hypertension, diabetes mellitus, dyslipidaemia, heart failure or left ventricular (LV) dysfunction (latest echocardiography (ECHO) or cardiac catheterisation), New York Heart Association (NYHA) class, previous history of stroke, TIA, or systemic embolism, renal disease, peripheral vascular disease, coronary artery disease, previous coronary angioplasty with or without stent, chronic obstructive pulmonary disease (COPD), smoking history, pulmonary hypertension (from latest ECHO or cardiac catheterisation), congenital aortic disease, previous cardiac surgery, history of atrial fibrillation and EuroSCORE II.

\section{Physical examination information}

Height $(\mathrm{cm})$, weight $(\mathrm{kg})$ and body surface area.

\section{Laboratory/tests}

We will obtain baseline haemoglobin $(\mathrm{g} / \mathrm{L})$, creatinine $(\mu \mathrm{mol} / \mathrm{L})$, baseline biomarkers of neuronal injury (S-100 $\beta$ and NSE) and a 12-lead ECG. 


\section{Imaging}

DW-MRI will be completed prior to the surgical date.

Neurocognitive tests

MMSE and MOCA will be carried out preoperatively.

\section{Intraoperative}

Intraoperative data collection will include total operative time (from skin incision to closure of the skin), total CPB time, total cross-clamp time, total circulatory arrest time, total ACP time, total cooling time, rewarming duration, nadir nasopharyngeal temperature, mean arterial systolic and diastolic blood pressure, nadir haemoglobin concentration $(\mathrm{g} / \mathrm{L})$, intraoperative RBC transfusion (units) and intraoperative inotrope or vasopressor use.

\section{Postoperative}

Postoperative data will include total ICU stay, total ventilation time, postoperative hospital stay, in-hospital mortality, delirium, reoperations, postoperative MI, inotropic support, seizure, development of renal failure, atrial fibrillation, postoperative stroke, transfusion, sepsis and pulmonary complications. Serum S-100 $\beta$ and NSE levels will be drawn postoperatively at $24-48$ hours. At postoperative day 4 ( \pm 3 days), the patient will undergo postoperative DW-MRI. On the day of discharge, the patient will undergo testing for stroke/TIA using the Modified Rankin Scale in addition to repeat neurological physical examination. Neurocognitive testing will also be carried out on the day of discharge, and at the surgical follow-up visit conducted at 1 to 3 months postoperatively, using MOCA and MMSE tests.

\section{Study Follow-up}

Subjects will be followed daily during their postoperative course in hospital. A phone call at postoperative day 30 will be conducted to assess for vital status, stroke and TIA. In addition, there will be a follow-up visit in 1 to 3 months postoperatively to reassess neurocognitive status.

\section{Study sample size and power}

In this trial, the primary endpoint is the number of patients with new radiologically significant ischaemic brain lesions on DW-MRI, comparing preoperative and postoperative imaging. In accordance with previous literature, we anticipate that about $50 \%$ of patients will develop new, radiologically significant ischaemic lesions in the axillary cannulation group (control group). ${ }^{5}$ Based on a consensus of investigators from surgery, anaesthesia, neuroradiology and critical care, we have determined that an absolute difference of $25 \%$ is an acceptable non-inferiority margin. Finally, we have set the power of this trial at $80 \%$ (for the $25 \%$ margin) and a one-sided Type I error of $5 \%$ (non-inferiority comparisons are one sided by definition). Under these assumptions and conditions, a sample size of 100 subjects (50 per group) is required. Finally, we factor in a $10 \%$ attrition rate to arrive at a final sample size of 110 . This sample size also gives us greater than $80 \%$ power to detect a difference of $45 \mathrm{~min}$ in total operative time between the axillary artery and innominate artery cannulation strategies.

\section{Data analysis}

The primary safety analysis is a non-inferiority comparison of the proportion of patients acquiring new severe brain lesions. The observed difference will be compared against the non-inferiority margin of $25 \%$ using a one-sided z-test. Since the intention-to-treat approach employed in superiority trials biases towards no difference, it is inappropriate in the non-inferiority trial where lack of difference is the goal. Therefore, the primary analysis will be a per-protocol analysis. The difference in proportions and $90 \%$ CIs (corresponds more closely to the one-sided test being used) will be reported. Given the non-inferiority design for the primary safety variable, non-inferiority comparisons of this outcome will use a one-sided alpha of 0.025 .

Our primary efficacy endpoint, total operative time, will be tested for superiority. Thus, the total operative time will be compared with a two-sided t-test (alpha 0.05), and the mean difference and 95\% CI will be reported.

Binary outcomes (30 day mortality, stroke/TIA, neurocognitive dysfunction, delirium, seizures, encephalopathy, adverse events, local complications) will be compared using a $\chi^{2}$ test or Fisher's exact test if expected counts are less than 5. For each outcome, the absolute risk difference and $95 \% \mathrm{CI}$ will be reported. The quantitative DW-MRI parameters will be compared by t-tests and mean differences with $95 \%$ CIs will be reported. Whether or not patients experienced none, a single or multiple new lesions will be compared by a $\chi^{2}$ test. The mean levels of postoperative biomarkers (S-100 $\beta$ and NSE) will be compared. The adjusted mean difference and $95 \%$ CI will be reported for each biomarker. Intraoperative characteristics will be compared with t-tests and mean differences with $95 \%$ CIs will be reported.

The $p$ values to be reported will not be corrected for multiple comparisons. We will finalise the statistical analysis plan prior to locking of the database.

\section{DISCUSSION}

Axillary artery cannulation for achieving ACP with moderate levels of hypothermia is the currently preferred approach for establishing cerebral perfusion during aortic surgery. We describe the first prospective, randomised controlled trial evaluating the safety and efficacy profile of ACP via cannulation of the innominate artery in comparison to axillary artery cannulation.

We selected new radiologically significant ischaemic brain lesions as defined by DW-MRI as our primary safety endpoint. DW-MRI has a sensitivity and specificity of $92 \%$ and $97 \%$, respectively, in detecting new ischaemic lesions. Identification of restriction in diffusion of water molecules suggests cerebral ischaemia. Normal tissue appears grey on DW-MRI due to the Brownian motion and diffusion of water molecules. Ischaemic tissue, however, due to the prevention of normal loss of MRI signal by restricted 
diffusion, appears bright white. These differences are apparent within 5 days of injury. ${ }^{25}$

DW-MRI as a modality to assess neurological injury has been validated in the transcatheter aortic valve implantation (TAVI) population, and more recently in aortic surgery studies and trials. These studies have demonstrated that the number of new ischaemic lesions, in addition to the size of these lesions together, is the most prognostically significant measure. ${ }^{23-25}$ Recent literature shows that the rate of new ischaemic brain lesions on DW-MRI after aortic surgery ranges from $40 \%$ to $60 \%{ }^{53031}$ A recent clinical trial in neonatal aortic arch surgery also employed DW-MRI as a surrogate of neuronal injury comparing ACP to DHCA. This non-inferiority trial used location, number of lesions and size of lesions to characterise severity of injury. ${ }^{5}$ We used these data to inform our primary endpoint.

By comparing DW-MRI preoperatively and postoperatively, we will be able to effectively quantify new, radiologically significant ischaemic brain lesions. Furthermore, given that DW-MRI is highly sensitive for ischaemic brain lesions, it also has an ability to detect 'silent' brain injury. Silent brain injury has been shown to be a risk factor for delayed neurological decline. ${ }^{25} 3233$ Retrospective studies evaluating innominate or axillary artery cannulation have primarily assessed clinical endpoints such as stroke or temporary neurological deficits. As such events are relatively rare, it is difficult to assess for true differences in outcomes between treatment modalities with the sample sizes available for aortic surgery. Thus, new, radiologically significant ischaemic brain lesions found on DW-MRI serve as a useful surrogate marker for risk of neurological injury. The high incidence of new ischaemic brain lesions post-aortic surgery allows us to efficiently assess our outcome with a relatively small sample size of 110 subjects.

In addition to imaging, this trial aims to assess neurological injury by comparing preoperative and postoperative levels of circulating biomarkers of neuronal injury, specifically NSE and S-100ß. There is extensive literature that shows that proteins that are synthesised by astroglial cells or neurons, and that cross the blood-brain barrier, can be correlated in peripheral blood with brain injury. ${ }^{34-37}$ $\mathrm{S}-100 \beta$ is a small dimeric cytosolic protein that exists in multiple forms, with the beta form noted to be highly specific for the central nervous system. ${ }^{34}$ NSE is a dimer found in neurons and belongs to a group of hydrolytic enzymes. It is also present in erythrocytes, platelets, plasmatic cells and lymphocytes, which is why it is present in peripheral blood at very low physiologic concentrations. ${ }^{34}$

Given that these biomarkers are present at levels from nil to very low physiologic concentrations at baseline, and S-100 $\beta$ especially is very sensitive for detection of intracranial pathology, elevations in these markers postoperatively will suggest neuronal injury secondary to the procedure. Collecting preoperative and postoperative samples will allow us to correlate serum levels with neuronal injury secondary to the aortic surgery specifically.
Furthermore, we will use two neurocognitive tests to detect neurocognitive decline postoperatively and will assess for differences between both cannulation strategies. Studies evaluating neuronal injury post-aortic surgery or TAVI have shown that neuronal injury does not always manifest overtly as stroke, but rather can present more subtly as neurocognitive decline. These features are often most prominent in those that have pre-existing neurological disease or vascular dementia. ${ }^{38}$ As a part of our neurocognitive testing, we have chosen to use both the MMSE and MOCA. These are commonly used cognitive screening tools in clinical practice. The MMSE is designed to assess language and memory while the MOCA is designed to detect mild to moderate cognitive impairment. It has been found to have high sensitivity and specificity for detecting mild cognitive impairment. Although more sophisticated neurocognitive testing does exist, due to time constraints of performing such testing, and the lack of generalisability with such tests, the MOCA and MMSE were chosen for ease of administration and reproducibility of results. Furthermore, MOCA and MMSE have been employed in a number of cardiac surgery studies evaluating neurocognitive decline. ${ }^{27-29} 38$

It is important to recognise that there are important limitations of this trial. We have limited this randomised controlled trial to elective procedures on the ascending aorta and proximal arch. Patients planned for total arch replacement are excluded. Secondarily, we are not studying patients undergoing aortic dissection or urgent/ emergent operations. Emergency operations such as for aortic dissection would introduce many confounding variables and significantly impact the ability to accurately assess the safety and efficacy of innominate artery cannulation in comparison to axillary artery cannulation. In addition, our follow-up period ends at 1 to 3 months postoperatively; therefore, the long-term outcomes of the different antegrade cerebral perfusion strategies will not be assessed. However, the catastrophic consequences of inadequate cerebral perfusion are usually apparent soon after surgery. Furthermore, our trial assumes competency of the operating aortic surgeon for establishing both cerebral protection strategies. Poor outcomes that may be linked to techniques by surgeons less familiar with either the innominate or axillary artery cannulation technique will not be known since only surgeons who routinely practice aortic surgery are participating in this study.

\section{ETHICS AND DISSEMINATION}

The protocol and consent form was initially approved at the lead site (St. Michael's Hospital Research Ethics Board; protocol 15-071) and subsequently by all other participating local research ethics boards (see online supplementary appendix 2). This study is being carried out in accordance with the current International Conference on Harmonisation Guideline for Good Clinical Practice, Tri-Council Policy Statement: Ethical Conduct for Research Involving Humans and applicable local laws 
and regulations. Protocol modifications will be communicated to each participating site and their respective research ethics committees. In addition, protocol amendments will be uploaded to ClinicalTrials.gov. As much data as possible will be contained in the published manuscript (and accompanying supplementary material), which is anticipated to occur in 2018 or 2019. There are no plans to provide public access to the participant-level database. All principal investigators will have access to the final cleaned data set. Site principal investigators will have access to their own site's data. All data sets will be password protected and any data to be disseminated will be de-identified.

\section{Patient Safety}

All subject-related information including case report forms, evaluation forms, reports etc. will be kept strictly confidential. All records will be kept in a secure, locked location and only research staff will have access to the records. Subjects will be identified only by means of a coded number specific to each subject. All computerised databases will identify subjects by numeric codes only and will be password protected.

An independent Data Safety and Monitoring Board (DSMB) composed of experts in cardiac surgery and perioperative care will be assembled to ensure patient safety, receive safety reports, provide feedback to the trial steering committee and ensure the study follows the highest ethical standards. The DSMB will be provided data on safety after enrolment of $1 / 3$ and $2 / 3$ of patients. The safety data will include all adverse events listed as primary and secondary outcomes. The DSMB will consider clinical and statistical significance, consistency of data over time, consistency of the direction of risk and benefitrisk ratios if there is consideration for recommendation for early trial discontinuation. In addition to receiving regular safety data reports, the DSMB will have the ability to request additional safety analyses or additional interim analyses and make any further recommendations to the steering committee about the safe conduct of the trial after considering all the available data and any new external data from relevant studies.

\section{CONCLUSION}

This randomised controlled trial (see online supplementary appendix 3) is essential to definitively determine adequate brain protection strategies for patients undergoing aortic surgery on the ascending aorta and proximal arch with the use of moderate hypothermia and unilateral antegrade cerebral perfusion. Innominate artery cannulation is rapidly gaining interest as an alternative to axillary artery cannulation; however, there are no highquality prospective data to inform whether or not the two strategies are similar with respect to cerebral protection. Innominate artery cannulation has the potential to decrease surgical times and reduce complications associated with an axillary approach such as brachial plexus injury, seroma formation and limb ischaemia. Decreased surgical times could lead to significant cost savings. The ACE CardioLink-3 trial will be the first randomised controlled trial designed to prospectively assess and compare the safety and efficacy of the innominate artery cannulation technique with the current standard of practice, axillary artery cannulation, for establishing ACP for patients having proximal aortic surgery. These data should aid surgeons in informed surgical decision making when considering cannulation techniques for aortic surgery.

\section{Author affiliations}

${ }^{1}$ Department of Internal Medicine, University of Toronto, Toronto, Ontario, Canada

${ }^{2}$ Division of Cardiac Surgery, Li Ka Shing Knowledge Institute of St. Michael's Hospital, Toronto, Ontario, Canada

${ }^{3}$ Department of Surgery, University of Toronto, Toronto, Ontario, Canada

${ }^{4}$ Division of Cardiac Surgery, London Health Sciences Center, University of Western Ontario, London, Ontario, Canada

${ }^{5}$ Division of Cardiovascular Surgery, Peter Munk Cardiac Centre, University Health Network, Toronto, Ontario, Canada

${ }^{6}$ Division of Cardiac Surgery, University of Alberta Hospital, University of Alberta, Edmonton, Alberta, Canada

${ }^{7}$ Division of Cardiac Surgery, Royal Jubilee Hospital, University of British Columbia, Victoria, British Columbia, Canada

${ }^{8}$ Division of Cardiac Surgery, Montreal Heart Institute, Université de Montréal, Montréal, Québec, Canada

${ }^{9}$ Division of Cardiac Surgery, Department of Surgery, Hamilton General Hospital, McMaster University, Hamilton, Ontario, Canada

${ }^{10}$ Applied Health Research Centre, Li Ka Shing Knowledge Institute of St. Michael's Hospital, Toronto, Ontario, Canada

${ }^{11}$ Dalla Lana School of Public Health, University of Toronto, Toronto, Ontario, Canada

${ }^{12}$ Division of Cardiac Surgery, Keenan Research Centre for Biomedical Science and Li Ka Shing Knowledge Institute of St. Michael's Hospital, Toronto, Ontario, Canada ${ }^{13}$ Division of Endocrinology \& Metabolism, Li Ka Shing Knowledge Institute of St. Michael's Hospital, Toronto, Ontario, Canada

${ }^{14}$ Department of Diagnostic and Therapeutic Neuroradiology, Keenan Research Centre for Biomedical Science of St. Michael's Hospital, University of Toronto, Toronto, Ontario, Canada

${ }^{15}$ Department of Medicine, University of Toronto, Toronto, Ontario, Canada

${ }^{16}$ Institute of Health Policy, Management and Evaluation, University of Toronto, Toronto, Ontario, Canada

${ }^{17}$ Leslie Dan Faculty of Pharmacy, University of Toronto, Toronto, Ontario, Canada

${ }^{18}$ Institute for Clinical Evaluative Sciences, University of Toronto, Toronto, Ontario, Canada

${ }^{19} \mathrm{Li}$ Ka Shing Centre for Healthcare Analytics Research and Training (LKS-CHART), St. Michael's Hospital, Toronto, Ontario, Canada

${ }^{20}$ Department of Anesthesia, Li Ka Shing Knowledge Institute of St. Michael's Hospital, Toronto, Ontario, Canada

${ }^{21}$ Department of Anesthesia, University of Toronto, Toronto, Ontario, Canada

${ }^{22}$ Department of Pharmacology and Toxicology, University of Toronto, Toronto, Ontario, Canada

Contributors VG, MDP, CDM and SV conceived the study and designed the study protocol. VG, MDP, CDM and SV significantly contributed to the planning of analyses of the data. VG, MDP, AG, CDM and SV drafted the manuscript. VG, MDP, MWAC, MO, RGGM, JB, IH, FVC, AG, JH, KET, ND, HT, TRM, DAL, AQ, MM, PJ, CDM and SV critically revised the manuscript for important intellectual content, reviewed and approved the final manuscript and agree to be accountable for all aspects of the work.

Competing interests MDP has received research grant support and speaker/ consulting honoraria from Edwards Lifesciences. MWAC has received speaker/ consulting honoraria from Medtronic, Canada, Edwards Lifesciences, Livanova and Symetis. The other authors have no conflicts to declare.

Patient consent Not obtained.

Provenance and peer review Not commissioned; externally peer reviewed. 
Data sharing statement All collected data will be accounted for in the published study. No unpublished data will be made available to any parties.

Open Access This is an Open Access article distributed in accordance with the Creative Commons Attribution Non Commercial (CC BY-NC 4.0) license, which permits others to distribute, remix, adapt, build upon this work non-commercially, and license their derivative works on different terms, provided the original work is properly cited and the use is non-commercial. See: http://creativecommons.org/ licenses/by-nc/4.0/

(c) Article author(s) (or their employer(s) unless otherwise stated in the text of the article) 2017. All rights reserved. No commercial use is permitted unless otherwise expressly granted.

\section{REFERENCES}

1. Elefteriades JA, Farkas EA. Thoracic aortic aneurysm clinically pertinent controversies and uncertainties. J Am Coll Cardiol 2010;55:841-57.

2. Patel HJ, Deeb GM. Ascending and arch aorta: pathology, natural history, and treatment. Circulation 2008;118:188-95.

3. Wolman RL, Nussmeier NA, Aggarwal A, et al. Cerebral injury after cardiac surgery: identification of a group at extraordinary risk. Multicenter Study of Perioperative Ischemia Research Group (McSPI) and the Ischemia Research Education Foundation (IREF) Investigators. Stroke 1999;30:514-22.

4. Boodhwani M, Andelfinger G, Leipsic J, et al. Canadian Cardiovascular Society position statement on the management of thoracic aortic disease. Can J Cardiol 2014;30:577-89.

5. Algra SO, Jansen NJ, van der Tweel I, et al. Neurological injury after neonatal cardiac surgery: a randomized, controlled trial of 2 perfusion techniques. Circulation 2014;129:224-33.

6. Hagl C, Ergin MA, Galla JD, et al. Neurologic outcome after ascending aorta-aortic arch operations: effect of brain protection technique in high-risk patients. J Thorac Cardiovasc Surg 2001;121:1107-21.

7. Appoo JJ, Bozinovski J, Chu MW, et al. Canadian Cardiovascular Society/Canadian Society of Cardiac Surgeons/Canadian Society for Vascular Surgery joint position statement on open and endovascular surgery for thoracic aortic disease. Can J Cardiol 2016;32:703-13.

8. Svensson LG, Blackstone EH, Rajeswaran J, et al. Does the arterial cannulation site for circulatory arrest influence stroke risk? Ann Thorac Surg 2004;78:1274-84.

9. Di Bartolomeo R, Pilato E, Pacini D, et al. Cerebral protection during surgery of the aortic arch. Multimed Man Cardiothorac Surg 2011;2011:mmcts.2010.004457.

10. Strauch JT, Spielvogel D, Lauten A, et al. Axillary artery cannulation: routine use in ascending aorta and aortic arch replacement. Ann Thorac Surg 2004;78:103--8.

11. Gulbins H, Pritisanac A, Ennker J. Axillary versus femoral cannulation for aortic surgery: enough evidence for a general recommendation? Ann Thorac Surg 2007;83:1219-24.

12. Reich DL, Uysal S, Ergin MA, et al. Retrograde cerebral perfusion as a method of neuroprotection during thoracic aortic surgery. Ann Thorac Surg 2001;72:1774-82.

13. Centofanti P, Barbero C, D'Agata F, et al. Neurologic and cognitive outcomes after aortic arch operation with hypothermic circulatory arrest. Surgery 2016;160:796-804.

14. Perreas K, Samanidis G, Thanopoulos A, et al. Antegrade or retrograde cerebral perfusion in ascending aorta and hemiarch surgery? A propensity-matched analysis. Ann Thorac Surg 2016;101:146-52.

15. Chu MW, Losenno KL, Gelinas JJ, et al. Innominate and axillary cannulation in aortic arch surgery provide similar neuroprotection. Can J Cardiol 2016;32:117-23.

16. Garg V, Tsirigotis DN, Dickson J, et al. Direct innominate artery cannulation for selective antegrade cerebral perfusion during deep hypothermic circulatory arrest in aortic surgery. J Thorac Cardiovasc Surg 2014;148:2920-4.

17. Sabik JF, Lytle BW, McCarthy PM, et al. Axillary artery: an alternative site of arterial cannulation for patients with extensive aortic and peripheral vascular disease. J Thorac Cardiovasc Surg 1995;109:885-90.

18. Sabik JF, Nemeh H, Lytle BW, et al. Cannulation of the axillary artery with a side graft reduces morbidity. Ann Thorac Surg 2004;77:1315-20.

19. Apostolakis E, Koletsis EN, Dedeilias $P$, et al. Antegrade versus retrograde cerebral perfusion in relation to postoperative complications following aortic arch surgery for acute aortic dissection type A. J Card Surg 2008;23:480-7.

20. Okita Y, Minatoya K, Tagusari O, et al. Prospective comparative study of brain protection in total aortic arch replacement: deep hypothermic circulatory arrest with retrograde cerebral perfusion or selective antegrade cerebral perfusion. Ann Thorac Surg 2001;72:72-9.

21. Bachet J, Guilmet D, Goudot B, et al. Antegrade cerebral perfusion with cold blood: a 13-year experience. Ann Thorac Surg 1999;67:1874-8.

22. Halkos ME, Kerendi F, Myung R, et al. Selective antegrade cerebral perfusion via right axillary artery cannulation reduces morbidity and mortality after proximal aortic surgery. J Thorac Cardiovasc Surg 2009;138:1081-9.

23. Thijs VN, Lansberg MG, Beaulieu C, et al. Is early ischemic lesion volume on diffusion-weighted imaging an independent predictor of stroke outcome? A multivariable analysis. Stroke 2000;31:2597-602.

24. Lövblad KO, Baird AE, Schlaug G, et al. Ischemic lesion volumes in acute stroke by diffusion-weighted magnetic resonance imaging correlate with clinical outcome. Ann Neurol 1997;42:164-70.

25. Meller SM, Baumbach A, Voros S, et al. Challenges in cardiac device innovation: is neuroimaging an appropriate endpoint? Consensus from the 2013 Yale-UCL Cardiac Device Innovation Summit. BMC Med 2013;11:257.

26. Chodosh EH, Foulkes MA, Kase CS, et al. Silent stroke in the NINCDS Stroke Data Bank. Neurology 1988;38:1674-9.

27. Baracchini C, Mazzalai F, Gruppo M, et al. Carotid endarterectomy protects elderly patients from cognitive decline: a prospective study. Surgery 2012;151:99-106.

28. Grace J, Nadler JD, White DA, et al. Folstein vs modified Mini-Menta State Examination in geriatric stroke. Stability, validity, and screening utility. Arch Neurol 1995;52:477-84

29. Dick JP, Guiloff RJ, Stewart A, et al. Mini-mental state examination in neurological patients. J Neurol Neurosurg Psychiatry 1984;47:496-9.

30. Kahlert P, Eggebrecht $H$, Jánosi RA, et al. Silent cerebral ischemia after thoracic endovascular aortic repair: a neuroimaging study. Ann Thorac Surg 2014;98:53-8.

31. Abdul-Jawad Altisent O, Ferreira-Gonzalez I, Marsal JR, et al. Neurological damage after transcatheter aortic valve implantation compared with surgical aortic valve replacement in intermediate risk patients. Clin Res Cardiol 2016;105:508-17.

32. Samim M, Agostoni P, Hendrikse J, et al. Embrella embolic deflection device for cerebral protection during transcatheter aortic valve replacement. J Thorac Cardiovasc Surg 2015;149:799-2e1-2.

33. Bolotin G, Huber $\mathrm{CH}$, Shani L, et al. Novel emboli protection system during cardiac surgery: a multi-center, randomized, clinical trial. Ann Thorac Surg 2014;98:1627-34.

34. Hajduková L, Sobek O, Prchalová D, et al. Biomarkers of brain damage: S100B and NSE concentrations in cerebrospinal fluid-a normative study. Biomed Res Int 2015;2015:1-7.

35. Di Battista AP, Buonora JE, Rhind SG, et al. Blood biomarkers in moderate-to-severe traumatic brain injury: potential utility of a multi-marker approach in characterizing outcome. Front Neurol 2015;6:110.

36. Romner B, Ingebrigtsen $\mathrm{T}$, Kongstad $\mathrm{P}$, et al. Traumatic brain damage: serum S-100 protein measurements related to neuroradiological findings. J Neurotrauma 2000;17:641-7.

37. Einav S, Kaufman N, Algur N, et al. Modeling serum biomarkers S100 beta and neuron-specific enolase as predictors of outcome after outof-hospital cardiac arrest: an aid to clinical decision making. J Am Coll Cardiol 2012;60:304-11.

38. Aggarwal A, Kean E. Comparison of the Folstein Mini Mental State Examination (MMSE) to the Montreal Cognitive Assessment (MoCA) as a cognitive screening tool in an inpatient rehabilitation setting Neuroscience \&amp; Medicine 2010;01:39-42. 